\title{
The post-translational phosphorylation and acetylation modification profile is not the determining factor in targeting endogenous stress-induced p53 to mitochondria
}

\author{
Cell Death and Differentiation (2005) 12, 197-200. doi:10.1038/sj.cdd.4401526 \\ Published online 19 November 2004
}

Dear Editor,

The pleiotropic apoptotic functions of p53 are critical for its powerful tumor suppressor activity. One mechanism is that p53 can act as transcription factor of proapoptotic target genes. We recently discovered a second transcriptionindependent mechanism that involves a rapid direct action of p53 protein at the mitochondria. ${ }^{1-3}$ In response to multiple death stimuli, a fraction of stabilized wild-type p53 rapidly translocates to mitochondria. Mitochondrial p53 physically interacts with critical $\mathrm{Bcl} 2$ family members and these interactions culminate in the permeabilization of the outer mitochondrial membrane, triggering the activation of effector caspases. In the first such interaction to be identified, we showed that mitochondrial wild-type p53 - but not tumor-derived DNA-binding mutants of p53 - forms complexes with antiapoptotic $\mathrm{BclXL}$ and $\mathrm{Bcl} 2$ proteins that (i) inhibit their membrane-protective actions ${ }^{2,4}$ and (ii) concomitantly induce Bak oligomerization and activation, which in turn permeabilizes the outer mitochondrial membrane, leading to rapid cytochrome $c$ release and caspase 3 activation. ${ }^{2}$ Recently, another complex between mitochondrial p53 and proapoptotic Bak was found in mitochondria of stressed cells. ${ }^{5}$ Importantly, the mitochondrial p53 program contributes to the physiologic p53 stress response in vivo. ${ }^{6}$ After $\gamma$-irradiation or intravenous etoposide, mitochondrial p53 accumulation occurs in radiosensitive organs such as thymus, spleen, testis and brain of normal mice, but not in radioresistant organs such as liver and kidney. Of note, mitochondrial p53 translocation is rapid (detectable by $30 \mathrm{~min}$ after stress in thymus and spleen) and triggers a first wave of caspase 3 activation and apoptosis, prior to the p53 transcriptional program. p53 target gene induction has a longer lag phase and coincides with a further increase in active caspase 3 levels. ${ }^{6}$

In response to genotoxic stress, p53 protein in vivo undergoes extensive post-translational phosphorylation of specific serine and threonine residues in the $\mathrm{N}$-terminal transactivation domain and acetylation of lysine residues in the C-terminal regulatory domain (reviewed by Appella and Anderson ${ }^{7}$ ). These modifications are believed to regulate $\mathrm{p53}$ accumulation and activation as a transcription factor and are mediated by kinase- and acetyltransferase-mediated signaling pathways that are partially stress-type specific. For example, ATM, Chk1, Chk2 and Cdk2 kinases mediate phosphorylation at Ser 15, Ser 20 and Ser 315 after DNA strand-breaking $\gamma$-ionizing radiation and topoisomerase inhibitors, while ATR, p38MAPK and CK2/FACT kinases mediate phosphorylation at Ser 15, Ser 37 and Ser 392 after basealtering UV light exposure. Phosphorylation of Ser 15, Thr 18, Ser 20 and Ser 37 stabilizes p53 at least in part due to interference with binding of the p53 E3 ligase MDM2. ${ }^{8}$ On the C-terminus, the acetyltransferase p300/CBP acetylates its major site Lys 382 and a minor site Lys 373 after $\gamma$-IR and UV in vivo, ${ }^{9-11}$ while PCAF acetylates a single site Lys 320 after UV exposure. $^{9,11}$ Interestingly, these modification reactions follow a specific sequential order regulated by an intramolecular phosphorylation-acetylation cascade. ${ }^{9} \mathrm{~N}$-terminal phosphorylation enhances p53 interaction with the acetyltransferases p300/CBP or PCAF, which promote acetylation of C-terminal Lys $382^{9,10}$ or Lys $320,{ }^{9}$ respectively, and activates sequencespecific DNA binding of p53. ${ }^{10}$ Since p300/CBP or PCAF are also transcriptional coactivators, this activates p53 transactivation and impairs p53 ubiquitination. ${ }^{12,13}$ Phosphorylation of Ser 315 and Ser 392 also contributes to regulating the tetramerization state of $\mathrm{p} 53^{12}$ and its ability to bind to cognate sequences in target gene promoters. ${ }^{14,15}$

Since post-translational p53 modification is so prominently linked to p53 accumulation and since p53 accumulation is linked to mitochondrial translocation, it has been speculated that a specific phosphorylation/acetylation profile is the determinant mitochondrial targeting signal for p53. To test this idea, we compared the modification patterns of nuclear and mitochondrial p53 protein after genotoxic stress. As expected, human ML-1 cells (wt p53) respond with marked p53 translocation to mitochondria after short-term genotoxic stress such as camptothecin or $\gamma$-IR (Figure 1a and data not shown). However, the profiles of mitochondrial and nuclear p53 proteins, when examined by two-dimensional isoelectric focusing/immunoblot analysis in $\mathrm{pH} 3-10$ and $\mathrm{pH}$ 5-8 gradients, were very similar (Figures $1 \mathrm{~b}, \mathrm{c}$ ). These data suggest that there are no major differences in the global modification patterns between mitochondrial and nuclear p53 in $\mathrm{ML}-1$ cells, although it leaves open the possibility that 
individual phosphorylation and/or acetylation sites on p53 might still be differentially modified in the two p53 protein pools. To test this possibility, we compared nuclear and mitochondrial p53 isolated from $\gamma$-irradiated or camptothecinstressed ML1 cells by examining multiple phosphorylation sites on Ser 6, Ser 9, Ser 15, Ser 20, Ser 33, Ser 37, Ser 46

a
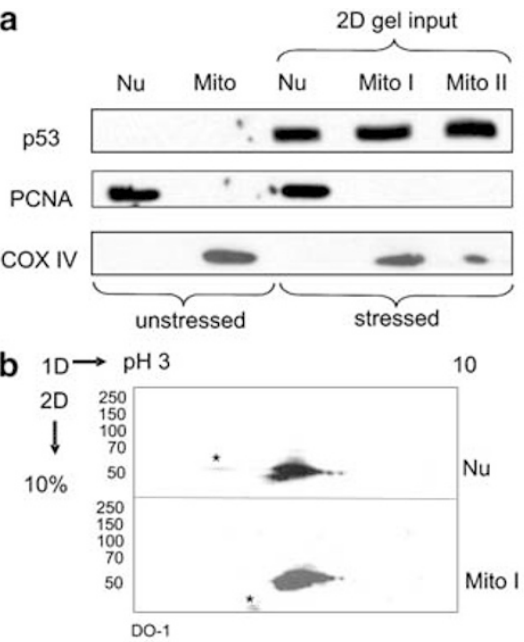

C

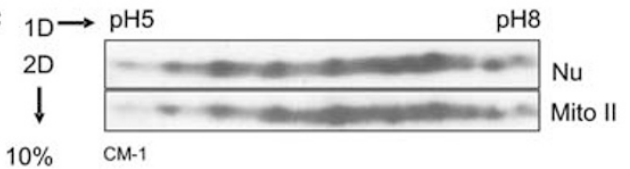

d

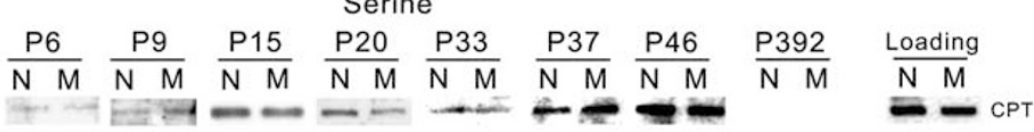

ML-1

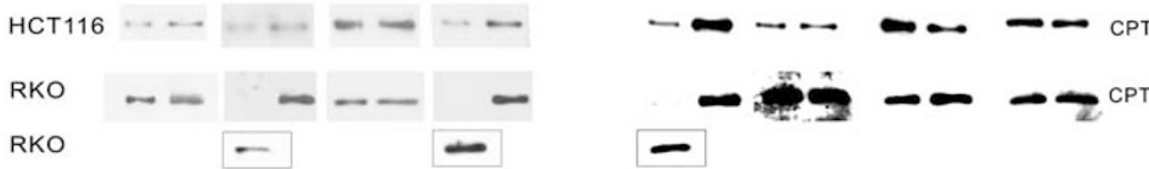

RKO
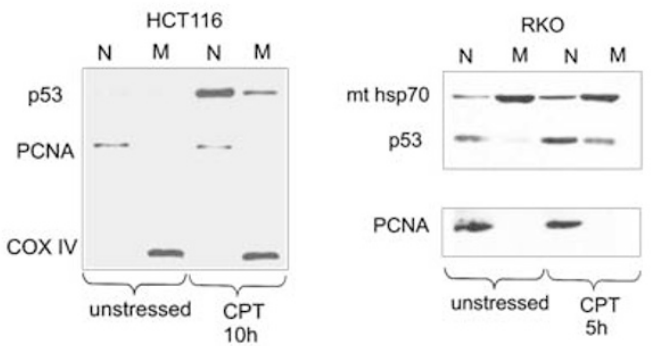

e

Serine

$\frac{P 9}{N M} \frac{P 15}{N M} \frac{P 20}{N M} \frac{P 33}{N M} \frac{P 37}{N M} \frac{P 46}{N M} \frac{P 392}{N M} \frac{\text { Loading }}{N M}$

$M L-1 \quad N M \quad N=-1-2-1 R$

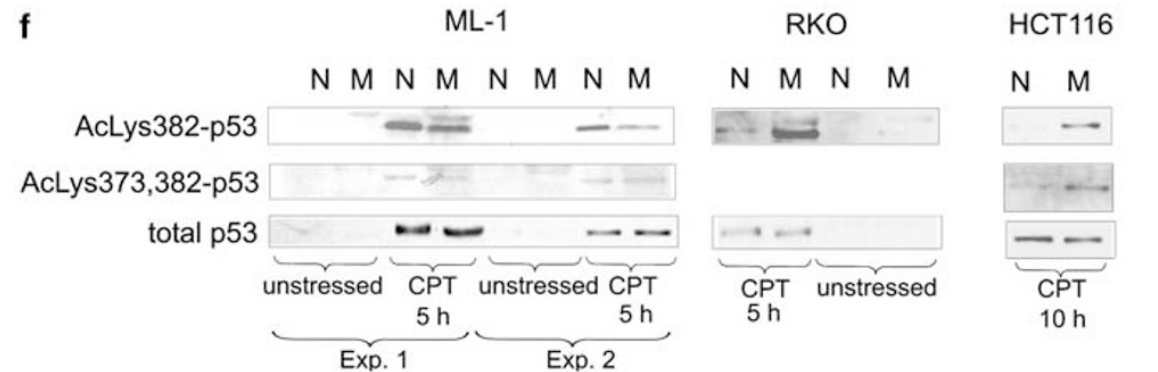


and Ser 392, using well-characterized phosphorylation sitespecific p53 antibodies. However, as seen in Figures 1d and $e$, no qualitative differences in phosphoserine patterns were detectable between the two p53 pools in ML-1 cells. In contrast, using two different p53 antibodies that specifically recognize AcLys 382 and AcLys 373/382, mitochondrial p53 reproducibly showed about a two-fold lower level of Lys 382 acetylation compared to nuclear p53 in ML-1 cells (Figure 1f). These data indicate that in ML-1 cells, which undergo a robust mitochondrial translocation response, the stress-induced nuclear and mitochondrial p53 protein pools do not differ in their global modification profiles. Thus, phosphorylation modifications do not seem to play a role in targeting p53 to mitochondria in these cells. Furthermore, the minor decrease in Lys 382 acetylation in mitochondrial p53 compared to nuclear p53 is unlikely to be responsible for its mitochondrial targeting, but rather reflects the fact that the coactivators $\mathrm{p300/CBP}$ and PCAF bind to the transcriptional pool of p53 in the nucleus after the two pools have separated. To further extend this result, we examined two additional (randomly chosen) wt p53expressing human cell lines, HCT116 (p53+/+) and RKO. These lines respond to DNA damage by camptothecin with clear but less robust mitochondrial translocation compared to ML-1 cells (Figure 1d bottom, compare stressed p53 $\mathrm{N} / \mathrm{M}$ signal ratios with those of $\mathrm{ML}-1$ in Figure 1a) and subsequent apoptosis that is p53 dependent (data not shown). As shown in Figure 1d, again no differences in phosphoserine patterns were detectable between the two p53 pools for Ser 6, Ser 9, Ser 15, Ser 20, Ser 46 and Ser 392 in HCT116 cells. Likewise, in RKO cells no phosphoserine differences were detected for Ser 6, Ser 15, Ser 46 and Ser 392. On the other hand, these cell lines showed a relative increase in phosphorylated Ser 37 in HCT116 cells and in phosphorylated Ser 9, Ser 20 and Ser 37 in RKO cells. Moreover, instead of a decrease in relative Lys 382 acetylation of mitochondrial p53 as seen in ML-1 cells, HCT116 and RKO both exhibited a relative increase in Lys 382 acetylation
(Figure 1f). Conversely, when HCT116 cells were induced to undergo mitochondrial p53 translocation by adriamycin or 5fluorouracil, the mitochondrial pool showed a lack of AcLys 382p53, while Ser15 phosphorylation did not show a difference compared to the nuclear pool. ${ }^{16}$ Thus, complex variability in overall modification profiles exists depending on the cell line and even the type of drug used within the same cell line. Importantly however, concerning mitochondrial p53, the data in sum clearly show a complete lack of correlation between the absence or presence of specific modifications and p53's ability to translocate to mitochondria. This strongly argues that none of these modifications are crucial for p53's mitochondrial targeting. In further support of this conclusion, none of these modifications appear to be crucial for p53's mitochondrial function based on our finding that nonmodified bacterial p53 is fully capable of mediating cytochrome $c$ release. ${ }^{2,5}$

A naturally occurring polymorphism at codon 72 of human p53 exerts additional control over the efficacy of mitochondrial translocation, in that the Arg 72 version of p53 has a greater ability to translocate to mitochondria than the Pro 72 version. ${ }^{17}$ In the engineered temperature-sensitive p53 Arg/ Pro SaOs2 model, nuclear export blockade by leptomycin $\mathrm{B}$ further suggested that mitochondrial p53 originates from the nucleus and utilizes p53 ubiquitination for mitochondrial targeting. ${ }^{17}$ Although attractive, this idea needs rigorous testing in physiologic cell systems. In our global 2D analysis on stressed ML1 cells, we saw no evidence of higher migrating ubiquitinated p53 variants in the mitochondrial p53 pool. Also, modification enzymes including MDM2 do not have a strict nuclear but also a cytoplasmic localization (and some can shuttle between the two compartments),${ }^{18}$ which does not rule out the possibility that endogenous mitochondrial p53 might originate directly from the cytoplasm. Taken together, while the identity of the mitochondrial p53 targeting signal is still open, data provided here fail to support phosphorylation and acetylation modifications, once thought to be prime candidates for targeting, to play a major role as mitochondrial localization signals.

Figure 1 The post-translational modification profile is not the determining factor in targeting endogenous stress-induced p53 to mitochondria. (a) ML1 cells were either left untreated or treated with $5 \mu \mathrm{M}$ camptothecin for $6 \mathrm{~h}$, followed by isolation of mitochondria with sucrose gradients. ${ }^{1}$ Two independent isolations (Mito I and Mito II) are shown that were subsequently used in 2D gel analysis (see $\mathbf{b}, \mathbf{c}$ ). Immunoblots on $10 \mu \mathrm{g}$ protein per lane for p53, PCNA (to verify lack of nuclear contamination) and COX IV (to verify mitochondrial enrichment). Nu Nuclear lysate. (b, c) Mitochondrial and nuclear p53 proteins examined by 2D isoelectric focusing/immunoblot analysis. Their global p53 dot profiles are very similar. Nuclei and mitochondria from ML1 cells treated with $5 \mu \mathrm{M}$ camptothecin for $6 \mathrm{~h}$ were prepared as in (a) and lysed in $9 \mathrm{M}$ urea, $2 \%$ NP-40, 3\% CHAPS, $70 \mathrm{mM}$ DTT and 2\% Ampholyte pH 8.5-10.5 (Sigma) containing $1 \mathrm{mM}$ PMSF, Protease Inhibitor Cocktail (Sigma) and Phosphatase Inhibitor Cocktail I (Sigma). Lysates were adjusted for equal quantities of mitochondrial and nuclear p53 by Western blots. The corresponding amounts of mitochondrial and nuclear proteins (between 25 and $35 \mu$ ) were mixed with $90 \mu \mathrm{l}$ rehydration buffer ( $8 \mathrm{M}$ urea, $4 \%$ CHAPS, $100 \mathrm{mM} \mathrm{DTT}, 0.001 \%$ Bromo phenol blue and $0.2 \%$ Ampholyte pH 5-8 or pH 3-10 (Sigma)) and applied to isoelectric focusing (IEF) strips (BioRad). After running the IEF strips at $4000 \mathrm{~V}$ for $17000 \mathrm{~V} \mathrm{~h}$ at $20^{\circ} \mathrm{C}$, they were equilibrated for 10 min in Buffer I ( $6 \mathrm{M}$ urea, $2 \%$ SDS, $0.05 \mathrm{M}$ Tris-HCl pH 8.8, 20\% glycerol, 2\% (w/v) DTT) followed by 10 min in Buffer II (6 M urea, 2\% SDS, $0.05 \mathrm{M} \mathrm{Tris-HCl} \mathrm{pH} \mathrm{8.8,}$ $20 \%$ glycerol, $2.5 \%$ iodoacetamide) and run on 10\% SDS-PAGE gels that were immunoblotted for p53 with DO1 (b) or CM1(c). (d, e) Comparison of post-translational phosphorylations at serine residues between mitochondrial and nuclear p53 protein. Nuclear and mitochondrial extracts from wt p53-expressing ML-1, HCT116 and RKO cells, each treated with $5 \mu \mathrm{M}$ camptothecin for 5,10 and $5 \mathrm{~h}$, respectively (d), or ML-1 cells harvested $4 \mathrm{~h}$ after $10 \mathrm{~Gy} \gamma-\mathrm{IR}$ (e) were prepared and quality controlled (a and d bottom; $10 \mu \mathrm{g}$ protein loaded per lane). All buffers used throughout processing contained phosphatase inhibitors (50 mM sodium fluoride, $2 \mathrm{mM}$ sodium orthovanadate). After immunoprecipitation with a 1:1 mixture of DO1-and 1801-conjugated Agarose beads, equal amounts of p53 were analyzed by immunoblots with well-characterized phosphorylation site-specific polyclonal p53 antibodies against the indicated residues (a generous gift from Y Taya). For pSer 15 and pSer 37 , lysates were directly probed with immunoblots. Equal loading of immunoprecipitated p53 is verified by CM-1 antibody. Note that nuclear p53 in HCT116 and RKO cells is phosphorylated at Ser 9, Ser 20 and Ser 37 and is acetylated at Lys 382, as revealed when blots are longer exposed (d, fourth row and data no shown). (f) Comparison of post-translational acetylation at Lys 382 and Lys $382 / 373$ between mitochondrial and nuclear p53 protein. Nuclear and mitochondrial extracts from wt p53-expressing ML1 (two independent experiments are shown as examples), RKO and HCT116 cells treated with $5 \mu \mathrm{M}$ camptothecin for the indicated times and prepared as in (d). Equal amounts of p53 (see row 'total p53') were immunoblotted with acetylation site-specific polyclonal p53 antibodies p53AcLys 382 (from Cell Signaling) and p53AcLys $373 / 382$ (from Upstate Biotechnology). All buffers contained deacetylase inhibitors (0.01 mM TSA and $5 \mathrm{mM}$ nicotinamide) throughout processing. Equal loading is verified by DO-1 antibody 


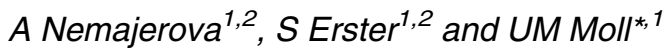

${ }^{1}$ Department of Pathology, Stony Brook University, Stony Brook, NY 11794 USA

2 These authors contributed equally to the paper

* Corresponding author: UM Moll, Department of Pathology, Stony Brook University, Stony Brook, NY 11794-8691, USA; Tel: 631-444-2459;

Fax: 631-444-3424; E-mail: umoll@ notes.cc.sunysb.edu

1. Marchenko ND, Zaika A and Moll UM (2000) J. Biol. Chem. 275: 16202-16212

2. Mihara $\mathrm{M}$ et al. (2003) Mol. Cell. 11: $577-590$

3. Sansome C et al. (2001) FEBS Lett. 488: 110-115

4. Petros AM et al. (2004) FEBS Lett. 559: 171-174
5. Leu Jl et al. (2004) Nat. Cell. Biol. 6: 443-450

6. Erster S et al. (2004) Mol. Cell. Biol. 24: 6728-6741

7. Appella E and Anderson CW (2001) Eur. J. Biochem. 268: 2764-2772

8. Shieh SY et al. (1997) Cell 91: 325-334

9. Sakaguchi K et al. (1998) Development 12: 2831-2841

10. Gu W and Roeder RG (1997) Cell 90: 595-606

11. Liu L et al. (1999) Mol. Cell. Biol. 19: 1202-1209

12. Sakaguchi $\mathrm{K}$ et al. (1997) Biochemistry 36: 10117-10124

13. Li M et al. (2002) J. Biol. Chem. 277: 50607-50611

14. Wang $Y$ and Prives $C$ (1995) Nature 376: 88-91

15. Hao M et al. (1996) J. Biol. Chem. 271: 29380-29385

16. Mahyar-Roemer M et al. (2004) Oncogene 23: 6226-6236

17. Dumont $P$ et al. (2003) Nat. Genet. 33: 357-365

18. Rotman $G$ and Shiloh $Y$ (1997) Cancer Surv. 29: 285-304 